\section{Assessing performances of Computer-Aided Diagnosis of breast cancer}

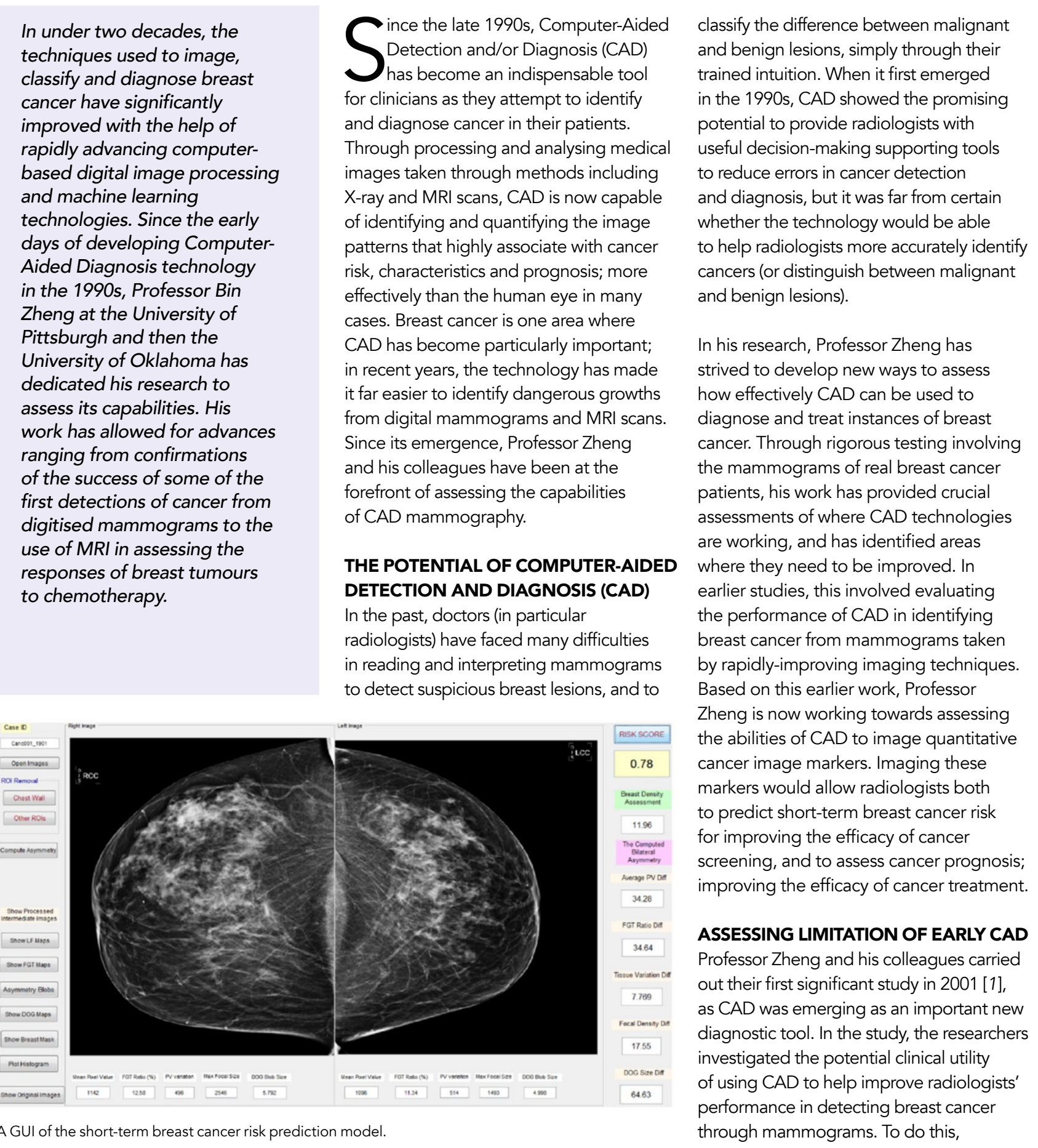

ince the late 1990s, Computer-Aided classify the difference between malignant $S_{\text {has become an indispensable tool }}^{\text {Detection andor }}$ for clinicians as they attempt to identify and diagnose cancer in their patients. Through processing and analysing medical Xages taken through methods incluading of identffing and quantifing the ipabege petterns that highly associte with agce iisk, characteristics and prognosiss more effectively than the human eye in many cases. Breast cancer is one area where CAD has become particularly important: in recent years, the technology has made It far easier to identify dangerous growths from digital mammograms and MR I scans. Since its emergence, Professor Zheng and his colleagues have been at the forefront of assessing the capabilities of CAD mammography

THE POTENTIAL OF COMPUTER-AIDED DETECTION AND DIAGNOSIS (CAD) adiologists) have faced many difficulties

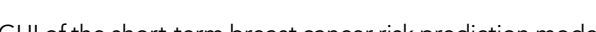

and benign lesions, simply through their trained intutition. When it tirst emerged in the 1990s, CAD showed the promising potential to provide radilogists with Wefl decision-making supporting tools and diagnosis, butit was far from cortan

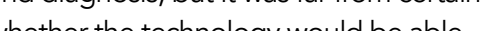
to help radiologists more accuratetlyidentiy cancers (or distinguish between malignant and benign lesions).

In his research, Professor Zheng has strived to develop new ways to assess how effectively $C A D$ can be used to diagnose and treat instances of breast cancer. Through higorous testing involving he mammograms of real breast cancer patients, his work has provided crucial assessments of where CAD technologies are working, and has identified areas where they need to be improved. In earlier studies, this involved evaluating he performance of $C A D$ in identifying by rapidly-improving imaging techniques Based on this earlier work, Professor Zheng is now working towards ssesesing the abilities of CAD to image quantitative cancer image markers. Imaging these markers would allow radiologists both to predict short-term breast cancer risk for improving the efficacy of cancer screening, and to assess cancer prognosis; improving the efficacy of cancer treatment

ASSESSING LMMTATION OF EARLY CAD Professor Zheng and his colleagues carried out their first significant study in 2001 [1], as CAD was emerging as an important new diagnostic tool. In the study, the researchers investigated the potential clinical utility of using CAD to help improve radiologists' through mammograms. To do this,

the team carried out a multi-mode based observer performance study involving from 209 women. While some of these patients had tested positiv for brest cancer according to severa reputable radiologists, the rest were confirmed to be cancer-free. For each screening Professor Zheng's team programmed the CAD scheme to four different levels of detection sensitivity and specificity (or false-positive rates) in detecting breast esions, which were represented by both soft tissue masses and micro-calcification clusters. Afterwards, eight radiologists independently read and interpreted this set of mammograms five times; firs without using $C A D$, and then using $C A D$ whour different performance levels. The researchers then quantified the rates of with the radiologists' conclusions in five reading modes.

The team found that when using highperformance CAD schemes with both high sensitivity and low false-positive rates, the radiologists' performance in detecting breast cancer from screening mammograms was significantly improved. At the same time, using CAD with lower performance levels, including either low sensitivity or higher false-positive rates, actually reduced radiologists performance. This laboratory study based finding was appraised by an editorial article of an expert in mammography [2], and has later been confirmed by a number of clinical studies (e.g., [3]). Thus, Professor theng's study revealed the importance CAD is used in clinical practice.

\section{ADAPTING TO ADVANCING CAD} TECHNOLOGY

ted great research interest to explore and develop new echnologies and approaches in the CAD research field. For example, Professor Zheng and his collaborators have not only converted the CAD schem from the previous screen-film based digitized mammograms to full-field digital mammograms (FFDM) [4]. The team has also been working towards developing and evaluating a number fovel approaches, which include but are not limited to multi-image based cueing methods $[6]$.
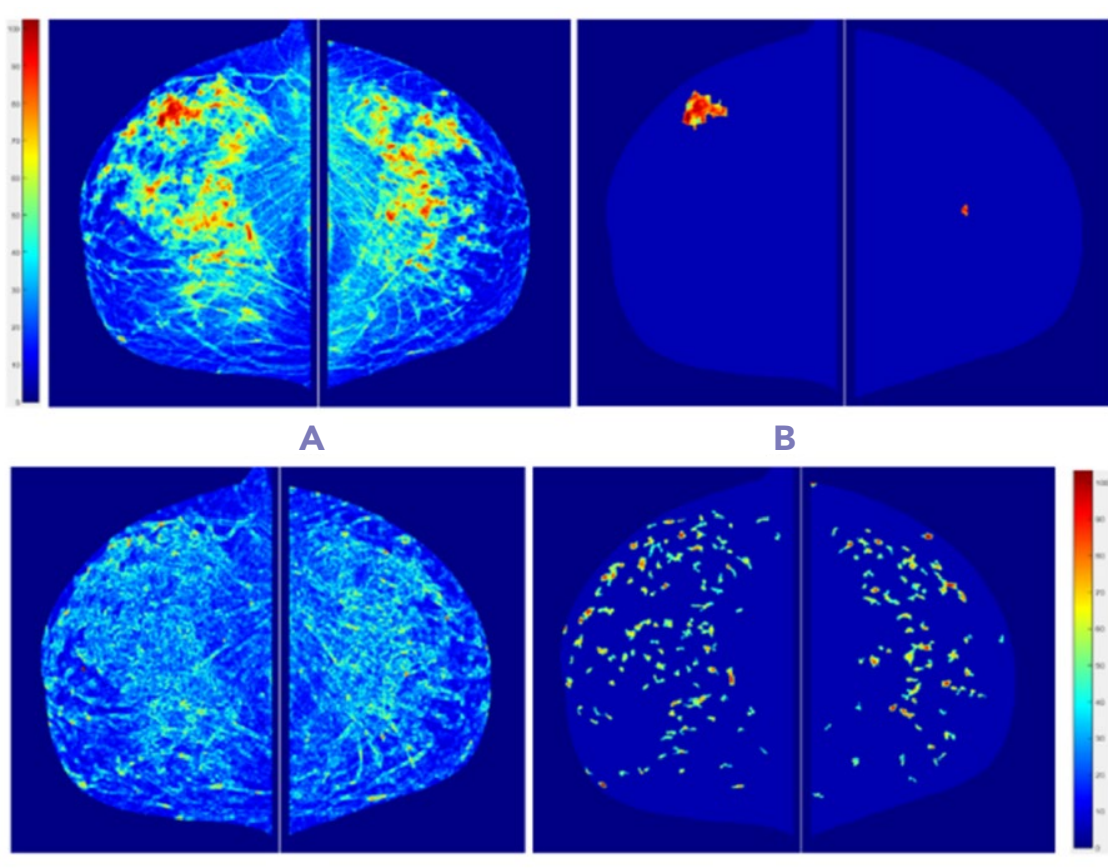
D

(B) detected focal density

Professor Zheng hopes that clinicians will soon be able to use CAD-generated markers to predict the risk of breast cancer in individual patients.

First, in developing a multi-view CAD scheme, the team proposed and applied a new narrow matching strip method, which significantly improved accuracy when matching two suspicious lesions detected in two view mammograms. As a resul, the new CAD scheme enables two views, without incering rates [5]. Second, the tean deve-positive a new case-based CAD scheme to predict the risk of a given case being positive for cancer, then fusing the case-based risk score with the conve
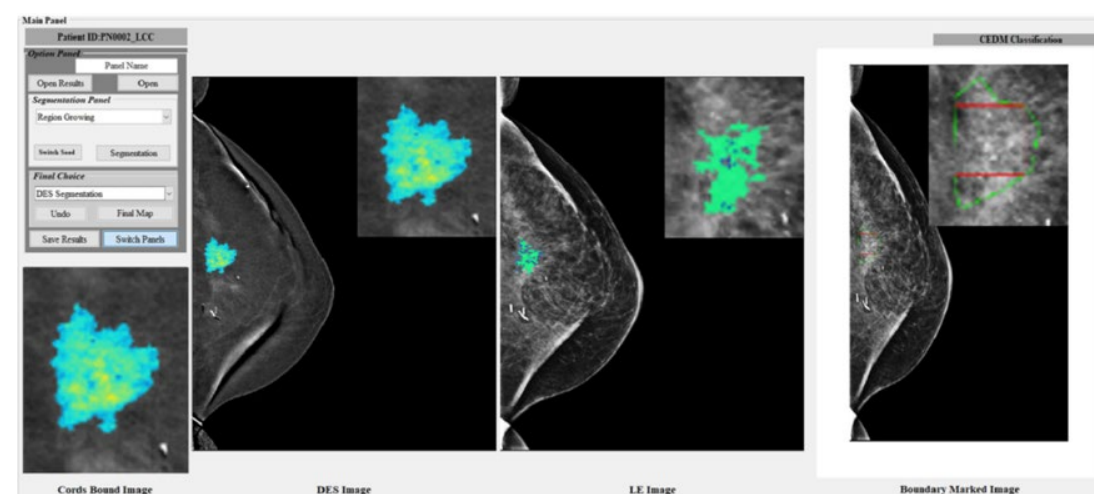

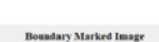

(or lesion) based score. This technique namely, an adaptive cueing method, to help detect more difficult or subtle positive rate [6].

In addition to detecting suspicious lesions, Professor Zheng and his team between malignant and benign bresst esions. For example, the team has performed pioneering work in applying contentbased image retrieval (CBIR) 
technology to develop CAD schemes usin a large, diverse and balanced reference regions of interest ( $\mathrm{RO}$ Is) that depict biopsyverified malignant and benign lesions. This CBIR-based CAD scheme not only achieves higher classification performance, but also pursues higher visual similarity aiming to increase radiologists' confidence in accepting CAD-generated classification results [7].

Recently, Professor Zheng and his team identified and investigated a new shortterm breast cancer risk factor, or prediction model, based on the quantitative analysis of bilateral mammographic density and tissue asymmetry. Several studies (i.e., [8], have demonstrated that using this new model had the potential to increase discriminatory power to predict short-term the efficacy of mammography screening.

\section{TRANSITION TO A}

\section{NEW IMAGING MODALITY}

mammograms (CEDM) have emerged as a promising new imaging modality in breast cancer imaging. The technique takes advantage of both DDDM, which provides high spatial resolution, and breast magnetic resonance imaging (MRl), which provides physiologically functional information w significantly fast scanning and low cost. However, the possibility of developing $C A D$ schemes of CEDM to assist radiologists in more accurately diagnosing suspicious
lesions has not been investigated before.

Professor Zheng and his collaborators in Mayor Clinic Arizona and Arizona State University have been working to The work reported in the ABME paper [9] presents the first fully-automated CAD scheme of CEDM to classify between malignant and benign breast lesions. The study results showed, firstly, that the segmentation of lesions from dual energy subtraction (DES) images was mu

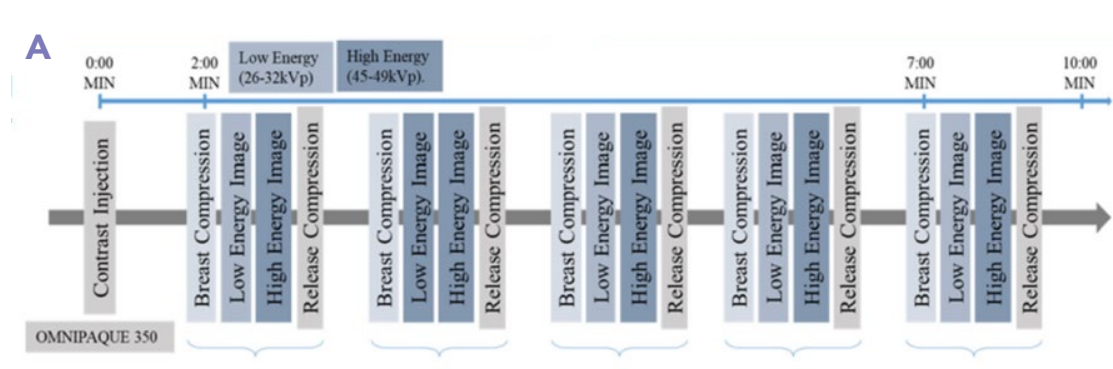

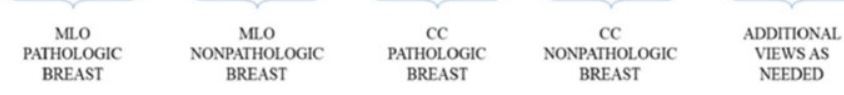
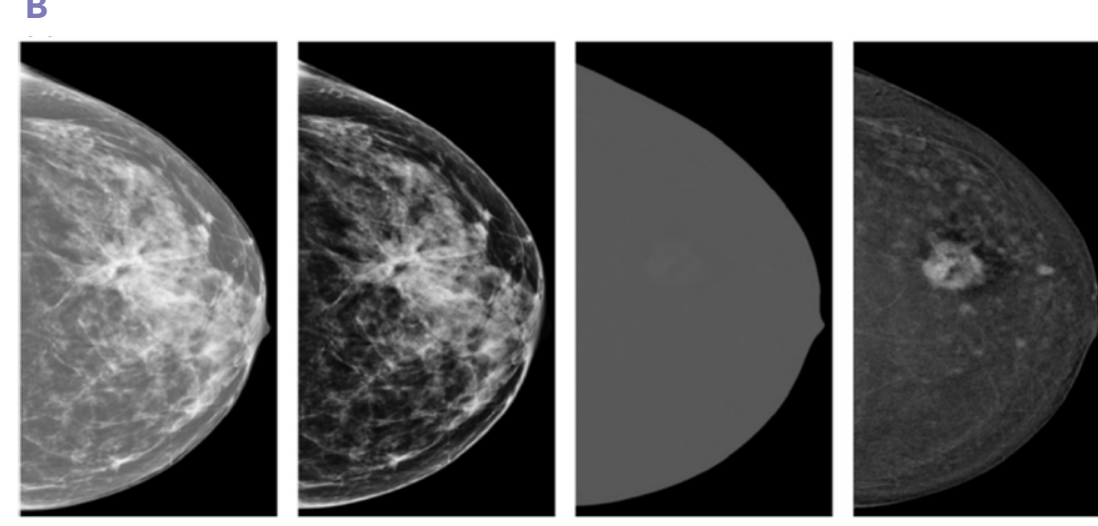

left to right: high energy image, low energy
he original and the adjusted window and leve for improving visibility, respective

easier and more accurate if lesions are lesion density heterogenever, DES lost which may be a disadvantage Finally, CAD yielded a significantly improved performance by mapping better lesion segmentation results from DES images to low-energy FFDM images. If the resuls can be further validated in future largescale studies, using CEDM and CAD will play an important role in reducing cancer diagnoses.

\section{GENERATING NEW}

\section{CAD-BASED IMAGE MARKERS}

Currently, Professor Zheng and a tean

of breast MR images, aiming to identfy new image markers for assessing the response of breast lesions to neoadjuva chemotherapy. For example, in a 2016 study, Professor Zheng and his colleagues used CAD to assess MR images of 151

Professor Zheng"s study revealed that even in the first years of its development, CAD already showed significant potential

to improve rates of a successful breast cancer diagnosis. chemotherapy for breast cancer; some to the treatment, and others displaying only a partial response. This time, CAD generated image markers by checking for bilateral asymmetry of dynamic contrast enhancement signals between The Tese makers were computed, and then selected to build a multi-feature, fusionbased machine learning model to distinguish between complete and partia usping $M$ ta

The study demonstrated that even with a large number of MR images, $C A D$ remained a reliable technique not only in identifying time-varying cancers from the images, but also for classifying between different responses of breast cancers to chemotherapy. This result reveals a promising potential for CAD to be used to generate quantitative cancer image markers in patients undergoing chemotherapy. With the insights provided by this latest development, Professor Zheng hopes that clinicians will soon individual patients, to classify between assess patient responses to chemo the 作 be able to use CAD-generated markers to predict the risk of breast cancer in malignant and benign tumours, and to

\section{Behind the Research}

\section{Professor Bin Zheng}

E: bin.zheng-1@ou.edu T: +14053253597 W: www.Medical-imaging.rccc.ou.edu/zheng (in www.linkedin.com/in/bin-zheng-49ba3232/ W: https://scholar.google.com/citations?user=FnKrwzOAAAAJ\&hl=en

\section{Research Objectives}

Professor Zheng's research aims to provide clinicians with "visual-aided" tools in cancer diagnosis, developing and validating
the computerised biomarkers extracted from the biomedical limages and electrical sirnals in order to help improve accuracy the computerised biomarkers extracted from the biomedical images and electrical signals in order to help improve accura
and reliability of predicting cancer risk, classifying suspicious lesions, assessing cancer prognosis and treatment efficacy.

Detail

101 David L. Boren Blvd, Suite 1001, Norman, OK 73072, USA

Bio
Bin Zheng received PhD from the University of Delaware. Currently, he is a professor of Electrical and Computer

the University of Oklahoma. His research interest is to develop and evaluate novel medical imaging informatics tools for disease risk prediction, early detection and prognosis assessment.

Funding

Cris well as Oklahoma Tobacco Settlement Endow and Charles Stephenson Cancer Center, University of Oklahoma.

\section{Collaborators}

Mayo Clinic Arizona

State University

Co-Pls of grant R01 CA197150:

- Dr Alan Hollingsworth in Mercy

- DrHong Liu at the University of Oklahoma

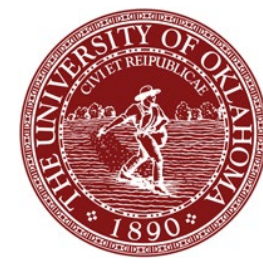

Personal Response

\section{What are your future phans for research in this area?}

Developing novel quantitative image markers usin CAD technology has demonstrated its potential to help Cinicians (i.e., radiologists, pathologists, oncologists

in sanger diagnosis and treatment. Thus, Professor Zhen and his research team will continue their research effort to explore and identify new image features from differen maging modalities, as well as develop and test new machine learning models that can optimally fuse multiple a hies higher discriminatory power in prediction of cancer risk, diagnosis of suspicious lesions, and assessment of

cancer prognosis and treatment efficacy.

\section{References}

[1] Zheng, B. Ganott, M.A. Britton, C.A. Hakim, C.M. Hardesty, L.A. Chang, T.S. Rockette, H.E. Gur, D. (2001). 'Soft-copy mammographic readings with different computer-assisted detection cuing environments:
preliminary findings'. Radiology, 221(3), 633-640.

[2] D'Orsl C. (2001). 'Computer-aided detection: There is no free lunch'. Radiology, 221:585-586.

[3] Fenton J, Abraham L, Taplin S, Geller B, Carney P, mammography practice'. Journal of National Cancer Institute, 103:1152-1161. [4] Zheng B, Sumkin JH, Zuley M, Lederman D, Wang $X$,
Gur D, (2012). 'Computer-aided detection of breast masses depicted on full-field digital mammograms:
a performance assessment'. British Journal of Radiology,
85:e153-161.

[5] Zheng B, Leader JK, Abrams GS, Lu AH, Wallace LP, aided detection scheme for breast masses'. Medical Physics, 33:3135-3143.

6] Wang X, Li L, XuW, Liu W, Lederman D, Zheng B, (2012). Improving performance of computer-aided detection of subtle breast masses using an adaptive cueing method. Z7.

77 Zheng B, Lu A, Hardesty LA, Sumkin JH, Hakim CM, similarity of breast masses for an interactive computersimilarity of breast masses for an interactive computer-
aided diagnosis environment'. Medical Physics, 33:111117

8] Wang X, Li L, Liu W, Xu W, Lederman D, Zheng B, (2012). 'An interactive system for computer-aided diagnosis of
breast masses'. Journal of Digital Imaging, 25:570-579.

9] Danala G, Patel B, A oei Figtal Imaging, Danala G, Patel B, Aghaei F, Heidari M, Li J, Wu
Zheng B, (2018). 'Classification of breast masses using a computer-aided diagnosis scheme of contrast Engineering, 46:1419-1431

[10] Aghaei, F. Tan, M. Hollingsworth, A.B. Zheng, B. (2016). 'Applying a new quantitative global breast MRI feature analysis scheme to assess tumor response to chemotherapy'. Journal of Magnetic Resonance
Imaging, 44(5), 1099-1106. 